\title{
On the Signless Laplacian Spectral Radius of Cacti
}

\author{
Mingzhu Chen, Bo Zhou*
}

\author{
School of Mathematical Sciences, South China Normal University, Guangzhou 510631, P. R. China \\ * Corresponding author's e-mail address: zhoubo@scnu.edu.cn \\ RECEIVED: October 8, 2016 * REVISED: February 24, 2017 * ACCEPTED: February 16, 2017
}

THIS PAPER IS DEDICATED TO PROF. NENAD TRINAJSTIĆ ON THE OCCASION OF HIS $80^{\text {TH }}$ BIRTHDAY

\begin{abstract}
A cactus is a connected graph in which any two cycles have at most one vertex in common. We determine the unique graphs with maximum signless Laplacian spectral radius in the class of cacti with given number of cycles (cut edges, respectively) as well as in the class of cacti with perfect matchings and given number of cycles.
\end{abstract}

Keywords: signless Laplacian spectral radius, graph, cactus, cycle, perfect matching, cut edge.

\section{INTRODUCTION}

A LL graphs in this paper are simple. Let $G$ be a graph on $n$ vertices with vertex set $V(G)$ and edge set $E(G)$. Let $A(G)$ be the adjacency matrix of $G$. The matrix $Q(G)=D(G)$ $+A(G)$ is called the signless Laplacian matrix of $G$, where $D(G)$ is a diagonal matrix of (vertex) degrees of $G$. It is well known that $Q(G)$ is a semi-definite matrix and thus its eigenvalues are all nonnegative. The signless Laplacian spectral radius of $G$ is the largest eigenvalue of $Q(G)$, denoted by $q(G)$. For more matrices associated to a graph, see the book of Janežič et al. ${ }^{[1]}$

If $G$ is connected, then $Q(G)$ is irreducible and by the Perron-Frobenius theorem, $q(G)$ has multiplicity one and there exists a unique positive unit eigenvector corresponding to $q(G)$, which is the Perron vector of $Q(G)$. The study of the signless Laplacian spectral radius of graphs has received much attention. ${ }^{[2-10]}$

A cactus is a connected graph in which every edge appears in at most one cycle, see, e.g. Ref. [11]. Note that trees and unicyclic graphs are cacti. The (adjacency) spectral radius and least eigenvalue of a cactus have been studied to some extent, ${ }^{[12-15]}$ and the distance spectral radius of a cactus was also studied. ${ }^{[16]} \mathrm{Li}_{\text {and }}$ Zhang ${ }^{[7]}$ determined the unique graphs with maximum signless Laplacian spectral radius in the class of cacti with given numbers of vertices and pendant vertices, and in the class of cacti with perfect matching and given number of vertices, respectively.
For $0 \leq k \leq[(n-1) / 2]$, let $\mathbb{C}(n, k)$ be the class of all cacti on $n$ vertices with $k$ cycles.

For $0 \leq k \leq n-3$, let $\mathbb{F}(n, k)$ be the class of all cacti on $n$ vertices with $k$ cut edges.

For $0 \leq k \leq n-1$, let $\mathbb{G}(n, k)$ be the class of all cacti on $2 n$ vertices with perfect matchings and $k$ cycles.

In this paper, we determine the unique cacti with maximum signless Laplacian spectral radius in $\mathbb{C}(n, k)$ for 0 $\leq k \leq[(n-1) / 2], \mathbb{F}(n, k)$ for $0 \leq k \leq n-3$, and $\mathbb{G}(n, k)$ for 0 $\leq k \leq n-1$, respectively.

The spectral radius was, long ago, put forward as a measure of molecular branching, ${ }^{[17]}$ while the Laplacian spectral radius was used for describing the shape and folding of DNA molecules. ${ }^{[18]}$ It is well known that the Laplacian and signless Laplacian spectra of bipartite graphs coincide. Thus, chemically interesting cases for signless Laplacian spectral radius are the fullerenes, fluoranthenes and other non-alternant conjugated species.

\section{PRELIMINARIES}

Let $G$ be a connected graph with $V(G)=\left\{v_{1}, \ldots, v_{n}\right\}$. The Perron vector of $Q(G)$ is the column vector $\mathbf{x}=\left(x_{v_{1}}, \ldots, x_{v_{2}}\right)^{\top}$, which can be considered as a function defined on $V(G)$ which maps vertex $v_{i}$ to $x_{v_{i}}$ for $i=1,2, \ldots, n$.

For an edge subset $F$ of a graph $G, G-F$ denotes the graph obtained from $G$ by deleting the edges in $F$, while for 
an edge subset $F^{\prime}$ of the complement of $G, G+F^{\prime}$ denotes the graph obtained from $G$ by adding the edges from $F^{\prime}$.

Denote by $C_{n}, P_{n}$ and $S_{n}$ the cycle, the path and the star on $n$ vertices, respectively.

For a graph $G$ with $u \in V(G), N_{G}(u)$ denotes the set of neighbors of $u$ in $G$, and the degree of $u$ in $G$ is $d_{G}(u)=$ $\left|N_{G}(u)\right|$.

The following two lemmas were proved in Refs. [19] and [20], respectively.

Lemma 2.1. Let $G$ be a connected graph with $u, v \in$ $V(G)$. Suppose that $w_{1}, \ldots, w_{s} \in N_{G}(v) \backslash\left(N_{G}(u) \cup\{u\}\right)$, where $s \geq$ 1. Let $\mathbf{x}=\left(x_{1}, \ldots, x_{n}\right)^{>}$be the Perron vector of $Q(G)$, and $G^{\prime}=G-$ $\left\{v w_{i}: i=1, \ldots, s\right\}+\left\{u w_{i}: i=1, \ldots, s\right\}$. If $x_{u} \geq x_{v}$, then $q\left(G^{\prime}\right)>q(G)$.

Lemma 2.2. Let $G$ be a connected graph and $e=u v a$ non-pendant edge of $G$. Suppose that $N_{G}(u) \cap N_{G}(v)=\emptyset$. Let $G^{\prime}$ be the graph obtained from $G-\{u v\}$ by identifying $u$ and $v$ into $u$, and adding a new pendant edge at $u$. Then, $q\left(G^{\prime}\right)>q(G)$.

The following lemma follows from the PerronFrobenius theorem, see, e.g., Ref. [5].

Lemma 2.3. Let $H$ be a proper subgraph of $a$ connected graph $G$. Then, $q(G)>q(H)$.

For $n \geq 3, k \geq 0$, and $0 \leq p \leq(n-2 k-1) / 2$, let $G_{n, k, p}$ be an $n$-vertex graph obtained by identifying a vertex of each of $k$ triangles, a vertex of each of $t$ paths $P_{2}$, and a terminal vertex of each of $p$ paths $P_{3}$, where $t=n-2 k-2 p-1$, see Figure 1.

Lemma 2.4. For $n \geq 3, k \geq 0$, and $0 \leq p \leq(n-2 k-1) / 2$, $q\left(G_{n, k, p}\right)$ is the largest root of the equation $f(x)=0$, where $f(x)$ $=x^{5}-(n-p+6) x^{4}+(6 n-6 p+10) x^{3}-(10 n+4 k-9 p+3) x^{2}+$ $(3 n+12 k) x-4 k$. In particular, $q\left(G_{n, k, 0}\right)$ is the largest root of the equation $x^{3}-(n+3) x^{2}+3 n x-4 k=0$, and for odd $n$,

$$
q\left(G_{n,(n-1) / 2,0}\right)=\frac{n+2+\sqrt{n^{2}-4 n+12}}{2} .
$$

Proof. The characteristic polynomial of $Q\left(G_{n, k, p}\right)$ was given in the proof of Lemma 4 in Ref. [8], from which the first part follows.

Let $g(x)=x^{3}-(n+3) x^{2}+3 n x-4 k$. If $p=0$, then it is easy to see that $f(x)=\left(x^{2}-3 x+1\right) g(x)$, which, together with the fact that $q\left(G_{n, k, 0}\right) \geq q\left(P_{3}\right)=3$, implies that $q\left(G_{n, k, 0}\right)$ is the largest root of the equation $g(x)=0$.

If $p=0, k=(n-1) / 2$, then $f(x)=\left(x^{2}-3 x+1\right)(x-1)\left[x^{2}\right.$ $-(n+2) x+2 n-2]$, and thus

$$
q\left(G_{n,(n-1) / 2,0}\right)=\frac{n+2+\sqrt{n^{2}-4 n+12}}{2} .
$$

For a cactus $G$ with at least one cycle, the deletion of all edges of $G$ on cycles results in a forest. A nontrivial

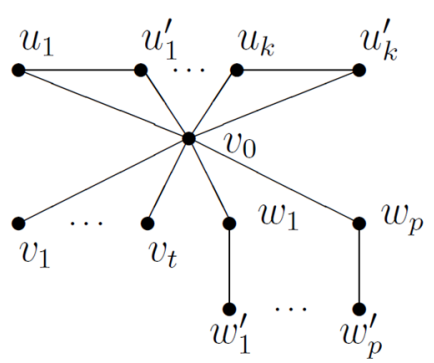

Figure 1. Graph $G_{n, k, p}$.

connected component of such a forest containing a unique vertex $v$ on some cycle is said to be a branch of $G$ at $v$. If $G$ is a tree, we also call it a branch at one of its vertices.

\section{SIGNLESS LAPLACIAN SPECTRAL RADIUS OF GRAPHS IN $\mathbb{C}(n, k)$}

Let $G_{n, k}=G_{n, k, 0}$. Note that $G_{n, 0}=S_{n}$.

Theorem 3.1. Let $G \in \mathbb{C}(n, k)$, where $0 \leq k \leq[(n-$ 1)/2] and $n \geq 3$. Then, $q(G) \leq q\left(G_{n, k}\right)$ with equality if and only if $G \cong G_{n, k}$, where $q\left(G_{n, k}\right)$ is the largest root of the equation $x^{3}-(n+3) x^{2}+3 n x-4 k=0$.

Proof. By Lemma 2.4, $q\left(G_{n, k}\right)$ is the largest root of the equation $x^{3}-(n+3) x^{2}+3 n x-4 k=0$. Let $G$ be a graph in $\mathbb{C}(n, k)$ with maximum signless Laplacian spectral radius. We need only to show that $G \cong G_{n, k}$.

Let $\mathbf{x}$ be the Perron vector of $Q(G)$.

Suppose that $k \geq 1$ and there is a cycle of length at least 4 . Let $v_{1} \ldots v_{r} v_{1}$ be such a cycle with length $r \geq 4$. Without loss of generality assume that $x_{v 1} \geq x_{v 2}$. Let $G^{\prime}=G-\left\{v_{2} v_{3}\right\}+\left\{v_{1} v_{3}\right\}$. Obviously, $G^{\prime} \in \mathbb{C}(n, k)$. By Lemma 2.1, we have $q\left(G^{0}\right)>q(G)$, a contradiction. Thus, if $k \geq 1$, then all cycles of $G$ are triangles. If $n=3$, then $k=1$ and thus the result follows. So let $n \geq 4$.

Claim 1. If $k \geq 2$, then any two triangles of $G$ have one common vertex.

Suppose that there are two disjoint triangles $T_{1}$ and $T_{2}$ in $G$. Then, there exists a unique shortest path $v_{1} \ldots v_{s}$ joining them, where $s \geq 2, v_{1} \in V\left(T_{1}\right), v_{s} \in V\left(T_{2}\right)$. If $s>2$, then $v_{i} \notin V\left(T_{1}\right) \cup V\left(T_{2}\right)$ for $2 \leq i \leq s-1$. Since $G$ is a cactus, any path joining $T_{1}$ and $T_{2}$ starts from $v_{1}$ and ends in $v_{s}$. We may assume that $x_{v 1} \geq x_{v s}$. Let $w_{1}$ and $w_{2}$ the neighbors of $v_{s}$ in $T_{2}$. Let $G^{\prime}=G-\left\{v_{s} w_{1}, v_{s} w_{2}\right\}+\left\{v_{1} w_{1}, v_{1} w_{2}\right\}$. Obviously, $G^{\prime} \in \mathbb{C}(n, k)$. By Lemma 2.1, we have $q\left(G^{\prime}\right)>q(G)$, a contradiction. This proves Claim 1.

Claim 2. If $k \geq 3$, then any three triangles of $G$ have exactly one common vertex.

Suppose that there are three triangles $T_{1}, T_{2}$ and $T_{3}$ in $G$ such that they have no common vertex. By Claim 1, the common vertices of $T_{1}$ and $T_{2}, T_{2}$ and $T_{3}$, and $T_{1}$ and $T_{3}$ induce 
another triangle having a common edge with $T_{1}$, a contradiction. Thus, Claim 2 follows.

By Claims 1 and 2, we have: if $k \geq 2$, then all triangles of $G$ have exactly one common vertex, which we denote by $v_{0}$. If $k=1$, choose $v_{0}$ such that $x_{v_{0}}$ is maximum among vertices with degree at least 3 on the unique triangle, and if $k=0$, choose $v_{0}$ to be a non-pendant vertex.

Claim 3. For $k \geq 1$, there is no branch of $G$ at a vertex different from $v_{0}$.

Suppose that there is a branch $T$ at vertex $v$ on some triangle $L$ of $G$ with $v \neq v_{0}$. Let $G^{\prime}=G-\left\{v w: w \in N_{T}(v)\right\}+$ $\left\{v_{0} w: w \in N_{T}(v)\right\}$ for $x_{v_{0}} \geq x_{v}$, and $G^{\prime}=G-\left\{v_{0} w: w \in N_{G}\left(v_{0}\right)\right.$ $\backslash V(L)\}+\left\{v w: w \in N_{G}\left(v_{0}\right) \backslash V(L)\right\}$ for $x_{v_{0}}<x_{v}$. In either case, $G^{\prime} \in \mathbb{C}(n, k)$, and by Lemma 2.1, we have $q\left(G^{\prime}\right)>q(G)$, a contradiction. Thus, Claim 3 follows.

If there is a branch $T$ of $G$ at $v_{0}$ (possibly $k=0$ and $G=$ $T$ ), then $T$ is a star with center $v_{0}$. Otherwise, suppose that there is a path $v_{0} v_{1} \ldots v_{s}$ in $T$, where $s \geq 2$. Let $G$ be the graph obtained from $G$ by deleting the edge $v_{0} v_{1}$, identifying $v_{0}$ and $v_{1}$ into $v_{0}$, and adding a new pendant edge to $v_{0}$. Obviously, $G^{\prime} \in \mathbb{C}(n, k)$. By Lemma 2.2, $q(G)<q\left(G^{\prime}\right)$, a contradiction. Now by Claims $1-3, G \cong G_{n, k}$.

Let $G$ be a cactus on $n \geq 3$ vertices. Let $k$ be the number of cycles of $G$, where $0 \leq k \leq[(n-1) / 2]$. By Theorem 3.1 and Lemma 2.3, $q(G) \leq q\left(G_{n, k}\right) \leq q\left(G_{n,[(n-1) / 2]}\right)$ with equalities if and only if $G \cong G_{n, k}$ and $k=[(n-1) / 2]$, i.e., $G \cong$ $G_{n,[(n-1) / 2]}$. Thus, $q(G) \leq q\left(G_{n,[(n-1) / 2]}\right)$ with equality if and only if $G \cong G_{n, k}$. By Theorem 3.1, we have $q\left(G_{n,[(n-1) / 2]}\right)=$ $\left(n+2+\sqrt{n^{2}-4 n+12}\right) / 2$ for odd $n$, and $q\left(G_{n,[(n-1) / 2]}\right)$ is the largest root of the equation $x^{3}-(n+3) x^{2}+3 n x-2 n+4$ $=0$ for even $n$.

\section{SIGNLESS LAPLACIAN SPECTRAL RADIUS OF GRAPHS IN $\mathbb{F}(n, k)$}

In view of the proofs of Lemmas 2.6 and 2.7 in Ref. [7], we deduce the following two lemmas.

Lemma 4.1. Let $Y$ be a connected graph with $u_{0} \in V(Y)$. For $m \geq 5$, let $G_{1}$ be the graph obtained by identifying $u_{0}$ and $a$ vertex of $C_{m}, G_{2}$ be the graph obtained by identifying $u_{0}$ and a vertex of each of $(m-1) / 2$ triangles for odd $m$, and $G_{3}$ be the graph obtained by identifying $u_{0}$, a vertex of each of $(m-4) / 2$ triangles, and a vertex of one quadrangle for even $m$, see Figure 2. Then, $q\left(G_{1}\right)<q\left(G_{2}\right)$ if $m$ is odd, and $q\left(G_{1}\right)<q\left(G_{3}\right)$ if $m$ is even.

Lemma 4.2. Let $Y$ be a connected graph with $u_{0} \in V$ $(Y)$. Let $G_{4}$ be the graph obtained by identifying $u_{0}$ and a vertex of each of two quadrangles, and $G_{5}$ be the graph obtained by identifying $u_{0}$ and a vertex of each of three triangles, see Figure 3. Then, $q\left(G_{4}\right)<q\left(G_{5}\right)$.

For $1 \leq r \leq \mathrm{n} / 2-1$, let $G_{n, r}^{\prime}$ be $n$-vertex graph obtained by identifying a vertex of each of $r-1$ triangles, a vertex of a quadrangle, and a vertex of each of $n-2 r-2$ paths $P_{2}$, see Figure 4 .

Lemma 4.3. For $n \geq 4$ and $1 \leq r \leq n / 2-1, q\left(G_{n, r}^{\prime}\right)$ is the largest root of the equation $f(x)=0$, where $f(x)=x^{5}-(n+6) x^{4}$ $+(7 n+7) x^{3}-(14 n+4 r-10) x^{2}+(6 n+16 r-16) x-8 r+8$

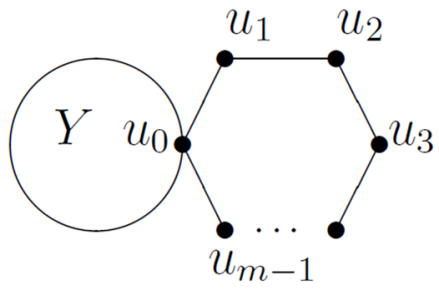

$G_{1}$

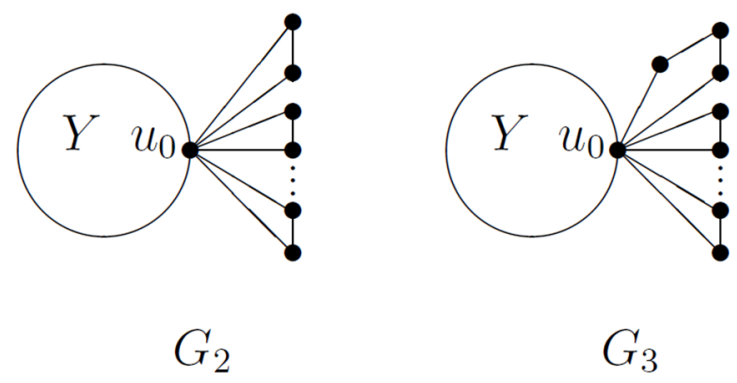

Figure 2. Graphs $G_{1}, G_{2}$, and $G_{3}$.

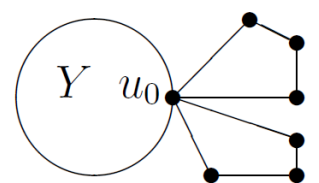

$G_{4}$

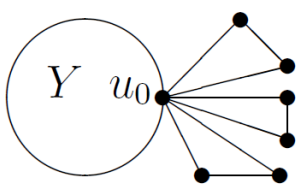

$G_{5}$
Figure 3. Graphs $G_{4}$, and $G_{5}$

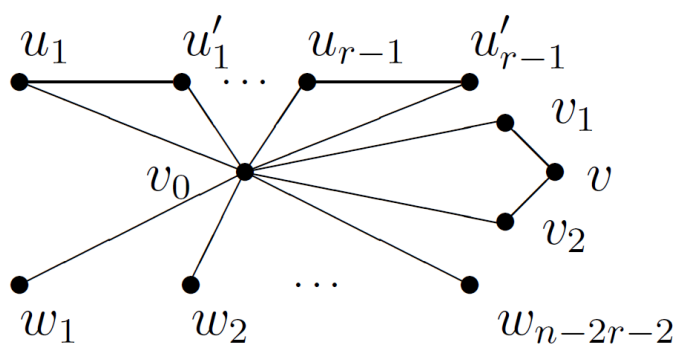

Figure 4. Graph $G_{n, r}^{\prime}$ 
Proof. Let $t=n-2 r-2$. Label the vertices of $G_{n, r}^{\prime}$ as $v_{0}, u_{1}, \ldots, u_{r-1}, u_{1}^{\prime}, \ldots, u_{r-1}^{\prime}, v_{1}, v_{2}, v, w_{1}, \ldots, w_{t}$, see Figure 4 .

Let $Q=Q\left(G_{n, r}^{\prime}\right), q=q\left(G_{n, r}^{\prime}\right)$ and $\mathbf{x}$ be the Perron vector of $Q$. By Lemma 2.3, $q \geq q\left(C_{4}\right)=4$.

Since $(q-2) x_{u_{i}}=x_{v_{0}}+x_{u_{i}}$ and $(q-2) x_{u_{i}}=x_{v_{0}}+x_{u_{i}}$ for $1 \leq i \leq r-1$, we have $x_{u_{i}}=x_{u_{i}}$ for $1 \leq i \leq r-1$ and thus $x_{u_{1}}=\ldots=x_{u_{r-1}}=x_{u_{1}}=\ldots=x_{u_{r-1}}$. Since $(q-1) x_{w_{i}}=x_{v_{0}}$ for $1 \leq i \leq t$, we have $x_{w_{1}}=x_{v_{t}}$. Thus,

$$
\begin{aligned}
& x_{v_{0}}-(q-3) x_{u_{1}}=0 \\
& x_{v_{0}}-(q-2) x_{v_{1}}+x_{v}=0 \\
& 2 x_{v_{1}}-(q-2) x_{v}=0 \\
& x_{v_{0}}-(q-1) x_{w_{1}}=0 \\
& (q-2 r-t) x_{v_{0}}-(2 r-2) x_{u_{1}}-2 x_{v_{1}}-t x_{w_{1}}=0
\end{aligned}
$$

Since $\mathbf{x} \neq \mathbf{0}$, we have $\left(x_{v_{0}}, x_{u_{1}}, x_{v_{1}}, x_{v}, x_{w_{1}}\right)^{\top} \neq 0$, and thus $\operatorname{det}(D)=0$, where

$$
D=\left(\begin{array}{ccccc}
1 & 3-q & 0 & 0 & 0 \\
1 & 0 & 2-q & 1 & 0 \\
0 & 0 & 2 & 2-q & 0 \\
1 & 0 & 0 & 0 & 1-q \\
q-2 r-t & 2-r & -2 & 0 & -t
\end{array}\right)
$$

By a direct calculation, we have $\operatorname{det}(D)=f(q)$.

Now it follows that $q$ is the largest root of the equation $f(x)=0$.

Theorem 4.1. Let $G \in \mathbb{F}(n, k)$, where $0 \leq k \leq n-3$.

(i) If $n-k$ is odd, then $q(\mathrm{G}) \leq q\left(G_{n,(n-k-1) / 2}\right)$ with equality if and only if $G \cong G_{n,(n-k-1) / 2}$, where $q\left(G_{n,(n-k-1) / 2}\right)$ is the largest root of the equation $x^{3}-(n+3) x^{2}+3 n x-2 n+$ $2 k+2=0$.

(ii) If $n-k$ is even, then $q(\mathrm{G}) \leq q\left(G_{n,(n-k-2) / 2}^{\prime}\right)$ with equality if and only if $G \cong G_{n,(n-k-2) / 2}^{\prime}$, where $q\left(G_{n,(n-k-2) / 2}^{\prime}\right)$ is the largest root of the equation $x^{5}-(n+6) x^{4}+(7 n+7) x^{3}-$ $(16 n-2 k-14) x^{2}+(14 n-8 k-32) x-4 n+4 k+16=0$.

Proof. By Lemmas 2.4 and 4.3 , if $n-k$ is odd, then $q\left(G_{n,(n-k-2) / 2}\right)$ is the largest root of the equation $x^{3}-(n$ $+3) x^{2}+3 n x-2 n+2 k+2=0$, and if $n-k$ is even, then $q\left(G_{n,(n-k-1) / 2)}^{\prime}\right)$ is the largest root of the equation $x^{5}-(n$ $+6) x^{4}+(7 n+7) x^{3}-(16 n-2 k-14) x^{2}+(14 n-8 k-32) x-$ $4 n+4 k+16=0$. Let $G$ be a graph in $\mathbb{F}(n, k)$ with maximum signless Laplacian spectral radius. We only need to show that is odd and is even.
The result is trivial for $n=3$ and for $n=4$ with $k=0$. Suppose $n \geq 4$ and $(n, k) \neq(4,0)$. By Lemma 2.3 and the fact that $q\left(C_{s}\right)=4$ for $s \geq 3$, we have $G \neq C_{n}$.

Since $k \leq n-3, G$ contains at least one cycle. By similar arguments as in Claims 1 and 2 in the proof of Theorem 3.1, if there are at least two cycles, then all cycles of $G$ have exactly one common vertex, denoted by $v_{0}$. If there is exactly one cycle in $G$, then choose $v_{0}$ such that the corresponding entry of the Perron vector of $Q(G)$ is maximum among vertices with degree at least 3 on the unique cycle. By similar arguments as in Claim 3 in the proof of Theorem 3.1, there is no branch of $G$ at a vertex different from $v_{0}$. If there is a branch $T$ of $G$ at $v_{0}$, then by Lemma 2.2, $T$ is a star with center $v_{0}$.

If $G$ contains a cycle of length at least 5 , then by Lemma 4.1, we may have a graph $G^{\prime} \in \mathbb{F}(n, k)$ (of the form $G_{2}$ or $G_{3}$ ) such that $q(G)<q\left(G^{\prime}\right)$, a contradiction. Thus, any cycle of $G$ has length 3 or 4 . If $G$ contains two quadrangles, then by Lemma 4.2, we may have a graph $G^{\prime} \in \mathbb{F}(n, k)$ (of the form $G_{5}$ ) such that $q(G)<q\left(G^{\prime}\right)$, a contradiction. Thus, $G$ has at most one quadrangle. Thus, $G \cong G_{n,(n-k-1) / 2}$ if $n-k$ is odd and $G \cong G_{n,(n-k-2) / 2}^{\prime}$ if $n-k$ is even.

\section{SIGNLESS LAPLACIAN SPECTRAL RADIUS OF GRAPHS IN $\mathbb{G}(n, k)$}

For a graph $G$, let $\Delta_{1}(G)$ be the maximum degree of $G$. If $d_{G}(u)=\Delta_{1}(G)$, where $u \in V(G)$, then let $\Delta_{2}(G)=\max \left\{d_{G}(v)\right.$ : $v \in V(G) \backslash\{u\}$.

The following lemma is a particular case of Theorem 4.2 in Ref. [21].

Lemma 5.1. Let $G$ be a graph on at least two vertices with $\Delta_{i}=\Delta_{i}(G)$ for $i=1,2$. Then,

$$
q(G) \leq \frac{\Delta_{1}+2 \Delta_{2}-1+\sqrt{\left(\Delta_{1}-2 \Delta_{2}-1\right)^{2}+8\left(\Delta_{1}-\Delta_{2}\right)}}{2} .
$$

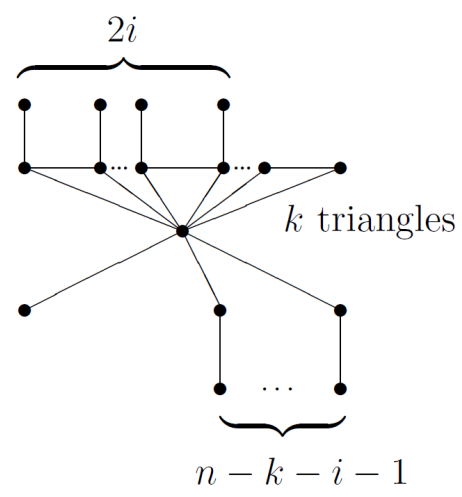

Figure 5. Graph $H_{n, k, i}$. 
The following lemma was given in Page 23 of Ref. [2] in plain text.

Lemma 5.2. Let $G$ be a connected graph on $n \geq 4$ vertices. Then, $q(G) \geq \Delta_{1}(G)+1$ with equality if and only if $G$ is the star $S_{n}$.

For $n \geq 2, k \geq 0,0 \leq i \leq k$, and $n-k-i-1 \geq 0$, let $H_{n, k, i}$ be the graph obtained from $G_{2 n-2 i, k, n-k-i-1}$ by attaching a pendant edge at each vertex with degree two of $i$ triangles, see Figure 5 .

Lemma 5.3. For $n \geq 3, k \geq 1,1 \leq i \leq k$ and $n-k-i-1$ $\geq 0$, we have $q\left(H_{n, k, i}\right)<q\left(G\left(H_{n, k, i-1}\right)\right)$.

Proof. By Lemma 5.1,

$$
\begin{aligned}
& \quad q\left(H_{n, k, i}\right) \leq \\
& \frac{(n+k-1)+6-1+\sqrt{(n+k-i-6-1)^{2}+8(n+k-1-3)}}{2}= \\
& \frac{(n+k-1)+5+\sqrt{(n+k-i-7)^{2}+8(n+k-1-3)}}{2} .
\end{aligned}
$$

By Lemma 5.2, $q\left(H_{n, k, i-1}\right)>n+k-i+2$. If $n+k-i \geq 6$, then $q\left(H_{n, k, i}\right) \leq n+k-i+2$, and thus $q\left(H_{n, k, i}\right)<q\left(G\left(H_{n, k, i-1}\right)\right.$.

Suppose that $n+k-i<6$. Since $k \geq i$ and $n \geq 3$, we have $n=3,4,5$. Since $k+i \leq n-1$ and $k \geq 1$, we have $k=1$, 2. Thus, $(n, k, i)=(3,1,1),(4,1,1),(4,2,1),(5,1,1),(5,2,2)$. By a direct calculation, we have

$$
\begin{aligned}
& q\left(H_{3,1,1}\right)=5.2361<5.3839=q\left(H_{3,1,0}\right), \\
& q\left(H_{4,1,1}\right)=5.7711<6.2860=q\left(H_{4,1,0}\right), \\
& q\left(H_{4,2,1}\right)=6.5741<7.2871=q\left(H_{4,2,0}\right), \\
& q\left(H_{5,1,1}\right)=6.5051<7.2261=q\left(H_{5,1,0}\right), \\
& q\left(H_{5,2,2}\right)=6.7359<7.4133=q\left(H_{5,2,1}\right) .
\end{aligned}
$$

Thus, the results follows.

Let $H_{n, k}=H_{n, k, 0}$.

Theorem 5.1. Let $G \in \mathbb{G}(n, k)$, where $n \geq 2$ and $0 \leq k$ $\leq n-1$. Then, $q(G) \leq q\left(H_{n, k}\right)$ with equality if and only if $G \cong$ $H_{n, k}$ where $q\left(H_{n, k}\right)$ is the largest root of the equation $x^{5}-(n+k+$ $7) x^{4}+(6 n+6 k+16) x^{3}-(11 n+13 k+12) x^{2}+(6 n+12 k) x-4 k=0$.

Proof. By Lemma 2.4, $q\left(H_{n, k}\right)$ is the largest root of the equation $x^{5}-(n+k+7) x^{4}+(6 n+6 k+16) x^{3}-(11 n+13 k+12) x^{2}$ $+(6 n+12 k) x-4 k=0$. Let $G$ be a graph in $\mathbb{G}(n, k)$ with maximum signless Laplacian spectral radius. We only need to show that $G \cong H_{n, k}$.

Let $\mathbf{x}$ be the Perron vector of $Q(G)$ and $M$ be a fixed perfect matching of $G$.

Suppose that $k \geq 1$ and there is a cycle $C=v_{1} \ldots v_{p} v_{1}$ of length $p \geq 4$. Without loss of generality assume that $x_{v 1}=$ $\min \left\{x_{v}: v \in V(C)\right\}$. Obviously, one of $v_{1} v_{2}$ and $v_{1} v_{p}$, say $v_{1} v_{2}$ is not in $M$. Let $G^{\prime}=G-\left\{v_{1} v_{2}\right\}+\left\{v_{p} v_{2}\right\}$. Then, $M$ is still a perfect matching of $G^{\prime}$, and $G^{\prime} \in \mathbb{G}(n, k)$. By Lemma 2.1, $q\left(G^{\prime}\right)>q(G)$, a contradiction. Thus, if $k \geq 1$, then all cycles of $G$ are triangles.
Claim 1. If $k \geq 2$, then any two triangles of $G$ have a common vertex.

Suppose that there are two disjoint triangles $T_{1}$ and $T_{2}$ in $G$. Then, there exists a unique shortest path $v_{1} \ldots v_{s}$ joining them, where $s \geq 2, v_{1} \in V\left(T_{1}\right), v_{s} \in V\left(T_{2}\right)$. If $s>2$, then $v_{i} \notin V\left(T_{1}\right)$ $\cup V\left(T_{2}\right)$ for $2 \leq i \leq s-1$. Note that any path joining $T_{1}$ and $T_{2}$ starts from $v_{1}$ and ends in $v_{s}$. We may assume that $x_{v s} \geq x_{v 1}$. Let $T_{1}=u v_{1} w$. Suppose that $u v_{1} \in M$. Then, $u w \notin M$ and that $w w^{\prime}$ $\in M$ for some vertex $w^{\prime} \in N_{G}(w) \backslash\left\{u, v_{1}\right\}$. If $x_{v 1} \geq x_{w}$, let $G^{\prime}=G-\{w y$ $\left.: y \in N_{G}(w) \backslash\left\{u, v_{1}\right\}\right\}+\left\{v_{1} y: y \in N_{G}(w) \backslash\left\{u, v_{1}\right\}\right\}$. Obviously, $M^{\prime}=$ $M-\left\{u v_{1}, w w^{\prime}\right\}+\left\{u w, v_{1} w^{\prime}\right\}$ is a perfect matching of $G^{\prime}$. Otherwise, let $G^{\prime}=G-\left\{v_{1} y: y \in N_{G}\left(v_{1}\right) \backslash\{u, w\}\right\}+\left\{w y: y \in N_{G}\left(v_{1}\right)\right.$ $\backslash\{u, w\}\}$. Obviously, $M$ is still a perfect matching of $G^{\prime}$. In either case, $G^{\prime} \in \mathbb{G}(n, k)$. By Lemma 2.1, $q\left(G^{\prime}\right)>q(G)$, a contradiction. Thus, $u v_{1} \notin M$. Similarly, $w v_{1} \notin M$. Let $G^{\prime}=G-\left\{u v_{1}, w v_{1}\right\}+$ $\left\{u v_{s}, w v_{s}\right\}$. Obviously, $G^{\prime} \in \mathbb{G}(n, k)$. By Lemma 2.1, $q\left(G^{\prime}\right)>q(G)$, a contradiction. Thus, Claim 1 follows.

By the same argument as in Theorem 3.1, we have

Claim 2. If $k \geq 3$, then any three triangles of $G$ have exactly one common vertex.

By Claims 1 and 2, we have: if $k \geq 2$, then all the triangles of $G$ have exactly one common vertex, denoted by $v_{0}$. If $k=1$, choose $v_{0}$ such that $x_{v_{0}}$ is maximum among vertices with degree at least 3 on the unique triangle, and if $k=0$, choose $v_{0}$ such that $x_{v 0}$ is maximum among vertices of $G$.

Claim 3. If $k \geq 1$ and there is a branch $T$ at vertex $v$ on some cycle of $G$, then $T$ is a tree consisting of pendant paths of length 2 and possibly one of length 1 at $v$. If $k=0$, then $G$ is a tree consisting of pendant paths of length 2 and possibly one of length 1 at $v_{0}$.

Let $u$ be a vertex furthest from $v$ in $T$ (possibly $k=0$, $v=v_{0}$, and $G=T$ ). Suppose that the distance between $v$ and $u$ in $T$ is at least 3. Since $G$ has perfect matchings, $u$ is a pendant vertex adjacent to a vertex $u^{\prime}$ of degree 2 . Let $w$ be the neighbor of $u^{\prime}$ different from $u$. Obviously, $w \neq v$. Let $G^{\prime}$ $=G-\left\{w u^{\prime}\right\}+\left\{v u^{\prime}\right\}$ if $x_{v} \geq x_{w}$ and $G^{\prime}=G-\left\{v v^{\prime}\right\}+\left\{w v^{\prime}\right\}$ otherwise, where $v^{\prime}$ is a neighbor of $v$ on some cycle such that $v v^{\prime} \notin M$. Obviously, $M$ is still a perfect matching of $G^{\prime}$, and $G^{\prime} \in \mathbb{G}(n, k)$. By Lemma 2.1, $q\left(G^{\prime}\right)>q(G)$, a contradiction. Thus, any vertex in $T$ is reachable from $v$ by a path of length at most 2. Now Claim 3 follows from the fact that $G$ has perfect matchings.

Claim 4. For $k \geq 1$ and a triangle $C=v_{0} u u_{1}$ in $G$, if there is a pendant edge at $u$, there is a pendant edge at $u_{1}$.

The claim is trivial for $k=1$. Suppose that $k \geq 2, u v$ is a pendant edge, and there is no pendant edge at $u_{1}$. Then, $u v, v_{0} u_{1} \in M$. If $x_{v_{0}} \geq x_{u}$, let $G^{\prime}=G-\{u v\}+\left\{v_{0} v\right\}$. Obviously, $M^{\prime}=M-\left\{u v, v_{0} u_{1}\right\}+\left\{u u_{1}, v_{0} v\right\}$ is a perfect matching of $G^{\prime}$. Otherwise, let $G^{\prime}=G-\left\{v_{0} y: y \in N_{G}\left(v_{0}\right) \backslash\left\{u, u_{1}\right\}\right\}+\{u y: y \in$ $\left.N_{G}\left(v_{0}\right) \backslash\left\{u, u_{1}\right\}\right\}$. Obviously, $M$ is still a perfect matching of $G^{\prime}$. In either case, $G^{\prime} \in \mathbb{G}(n, k)$. By Lemma 2.1, $q\left(G^{\prime}\right)>q(G)$, a contradiction. Thus, Claim 4 follows. 
Claim 5. For $k \geq 1$, there is no pendant path of length 2 in $G$ at a vertex different from $v_{0}$.

Suppose that $v_{0} u u_{1}$ is a triangle in $G$ and there is a pendant path $u v w$ at $u$. Then, $v w \in M$. If $x_{v 0} \geq x_{u}$, let $G^{\prime}=G$ $\{u v\}+\left\{v_{0} v\right\}$. Suppose that $x_{v 0}<x_{u}$. Since $G$ has perfect matchings, we have by Claim 4 that any edge on a triangle incident to $v_{0}$ is not in $M$, implying that there is a branch, say $T_{0}$ at $v_{0}$. Then, by our choice of $v_{0}$, we have $k \geq 2$, and for any vertex $y \in N_{G}\left(v_{0}\right) \backslash\left(\left\{u, u_{1}\right\} \cup \vee\left(T_{0}\right)\right), v_{0} y / \in M$. Let $G^{\prime}=$ $G-\left\{v_{0} y: y \in N_{G}\left(v_{0}\right) \backslash\left(\left\{u, u_{1}\right\} \cup V\left(T_{0}\right)\right)\right\}+\left\{u y: y \in N_{G}\left(v_{0}\right) \backslash\right.$ $\left.\left(\left\{u, u_{1}\right\} \cup V\left(T_{0}\right)\right)\right\}$. Obviously, $M$ is still a perfect matching of $G^{\prime}$ and $G^{\prime} \in \mathbb{G}(n, k)$ whether $x_{v 0} \geq x_{u}$ or not. By Lemma 2.1, we have $q\left(G^{\prime}\right)>q(G)$ in either case, a contradiction. Thus, Claim 5 follows.

By Claims $1-5, G \cong H_{n, k, i}$ for some $i$ with $0 \leq i \leq k$. If $n$ $=2, k \geq 1$ the result is trivial. If $n \geq 3, k \geq 1$, then by Lemma $5.3, G \cong H_{n, k}$.

Note that $\mathbb{G}(n, 0)$ is the set of trees of order $2 n$ with perfect matching. Thus, the case $k=0$ in Theorem 5.1 has been studied in Ref. [22].

\section{CONCLUSION}

In this paper, we investigate the extremal problems for signless Laplacian spectral radius of cacti. We determine the unique graphs with maximum signless Laplacian spectral radius of graphs in the class of cacti with given number of cycles (cut edges, respectively) and in the class of cacti with perfect matchings and given number of cycles. The extremal graphs (except the case for trees) are not bipartite.

\section{REFERENCES}

[1] D. Janežič, A. Miličević, S. Nikolić, N. Trinajstić, Graph-Theoretical Matrices in Chemistry, CRC Press, Boca Raton, 2015.
[2] D. Cvetković, P. Rowlinson, S. K. Simić, Publ. Inst. Math. 2007, 81, 11.

[3] D. Cvetković, S. K. Simić, Linear Algebra Appl. 2010, 432, 2257.

[4] D. Cvetković, P. Rowlinson, S. K. Simić, Linear Algebra Appl. 2007, 423, 155.

[5] Y. Z. Fan, D. Yang, Graphs Combin. 2009, 25, 35.

[6] P. Hansen, C. Lucas, Linear Algebra Appl. 2010, 432, 3319.

[7] S. Li, M. Zhang, Linear Algebra Appl. 2012, 436, 4400.

[8] H. Lin, B. Zhou, Linear Multilinear Algebra 2015, 63, 377.

[9] C. S. Oliveir, L. S. de Lima, N. M. M. de Abreu, P. Hansen, Discrete Appl. Math. 2010, 158, 355.

[10] B. Zhou, Linear Algebra Appl. 2010, 432, 566.

[11] B. Zmazek, J. Zerovnik, Croat. Chem. Acta 2013, 76, 137.

[12] B. Borovićanin, M. Petrović, Publ. Inst. Math. 2006, 79 (93), 13.

[13] Z. Huang, H. Deng, S. K. Simić, Appl. Anal. Discrete Math. 2011, 5, 14.

[14] M. Petrović, T. Aleksić, V. Simić, Linear Algebra Appl. 2011, 435, 2357.

[15] R. Xing, B. Zhou, Linear Algebra Appl. 2013, 438, 2256.

[16] S. S. Bose, M. Nath, S. Paul, Linear Algebra Appl. 2012, 437, 2128.

[17] D. M. Cvetković, I. Gutman, Croat. Chem. Acta 1977, 49, 115.

[18] M. Randić, M. Vračko, M. Novič, in QSPR/QSAR Studies by Molecular Descriptors (Ed.: M.V. Diudea), Nova Science, New York, 2001, pp. 147-211.

[19] Y. Hong, X.D. Zhang, Discrete Math. 2005, 296, 187.

[20] S. G. Guo, Discrete Math. 2008, 308, 4608.

[21] X. Duan, B. Zhou, Linear Algebra Appl. 2013, 439, 2961.

[22] J. M. Guo, Linear Algebra Appl. 2003, 368, 379. 